\title{
A Rigorous Analysis of a Phase-Locked Oscillator Under Injection
}

\author{
Chien-Jung Li, Member, IEEE, Chieh-Hsun Hsiao, Fu-Kang Wang, \\ Tzyy-Sheng Horng, Senior Member, IEEE, and Kang-Chun Peng, Member, IEEE
}

\begin{abstract}
This study presents injection-pulling effects on a local oscillator (LO) for wireless applications. A discrete-time analysis is provided to predict output spectra of the LO pulled by a sinusoidal and angle-modulated injection signal. A phase-locked loop synthesizer with an injection signal is analyzed in frequency domain to account for the inherent bandpass filtering on the injection signal. In addition, a phase noise model is developed by using the proposed frequency-domain approach to characterize the overall phase noise of a phase-locked oscillator under injection. Comparison between theoretical predictions and experimental results shows excellent agreement.
\end{abstract}

Index Terms-Injection locking, injection pulling, local oscillator (LO) pulling, phase-locked loop (PLL).

\section{INTRODUCTION}

A LOCAL oscillator (LO) is a crucial component in an RF transceiver to provide a desired pure sinusoidal signal for modulation/demodulation or up/down frequency conversion. It is usually a phase-locked oscillator (PLO) that uses a phaselocked loop (PLL) to synchronize a voltage-controlled oscillator (VCO) to a reference frequency source. As depicted in Fig. 1, a PLO contains a phase and frequency detector (PFD), a charge pump (CP), a loop filter, a VCO, and a frequency divider in the feedback path. Precise control of the phase, and thus frequency, of the VCO is achieved through the feedback action of the loop. Owing to the nature of oscillators, a PLO is subject to interference, which often causes frequency pulling and hence degrades the spectral purity. Generally speaking, the interference comes from various sources, including power-amplified signals and spurious signals, and is injected into a VCO of a PLO via a parasitic coupling path [1], [2].

In the early days, Adler [3] and many other authors [4]-[7] studied the behavior of an oscillator under injection of an independent sinusoidal signal. In [3], the well-known Adler's equa-

Manuscript received August 09, 2009; revised December 28, 2009. First published April 01, 2010; current version published May 12, 2010. This work was supported in part by the National Science Council, Taiwan, under Grant 97-2221-E-110-035-MY3 and Grant 97-2628-E-110-041-MY3, and by the Department of Industrial Technology, Taiwan, under Grant 97-EC-17-A-01-S1-104.

C.-J. Li, C.-H. Hsaio, F.-K. Wang, and T.-S. Horng are with the Department of Electrical Engineering, National Sun Yat-Sen University, Kaohsiung 804, Taiwan (e-mail: m9131624@student.nsysu.edu.tw; jason@ee.nsysu.edu.tw).

K.-C. Peng is with the Department of Computer and Communication Engineering, National Kaohsiung First University of Science and Technology, Kaohsiung 811, Taiwan (e-mail: peterpkg@ccms.nkfust.edu.tw).

Color versions of one or more of the figures in this paper are available online at http://ieeexplore.ieee.org.

Digital Object Identifier 10.1109/TMTT.2010.2042898

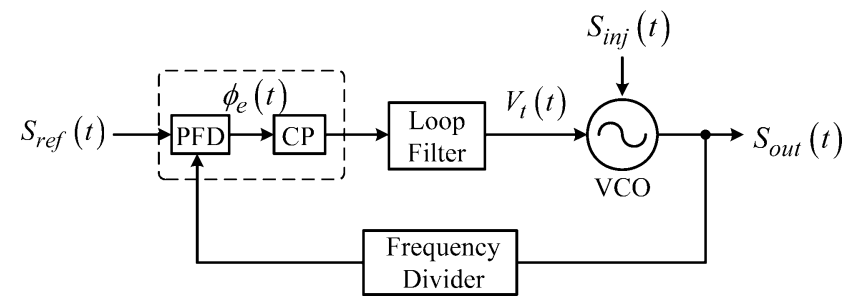

Fig. 1. Block diagram of a PLO under injection.

tion was given to account for the injection-locking phenomena in a free-running oscillator. Recently, there have been many approaches presented in the literature with prediction of the frequency-pulling effects. Mirzaei et al.[8] used a hard-limiter transconductor and an $R L C$ circuit to model an $L C$-tank oscillator under the influence of injection. On the basis of [8], Heidari and Abidi [9] presented a behavior model simulation to predict the frequency-pulling effects on a differential $L C$-tank oscillator and an outphasing wireless transmitter. Razavi [10] considered a PLO under sinusoidal injection and studied the resultant frequency-pulling effects in an intensive manner. In an effort to achieve an efficient prediction by computer, Lai and Roychowdhury [11], [12] developed a nonlinear phase-domain macromodel by means of a perturbation projection vector method to predict injection pulling in $L C$-tank and ring oscillators as well as in PLOs. Based on [11], Maffezzoni and D' Amore [13] presented a hybrid numerical-analytical approach to evaluate frequency pulling in oscillators with small-signal injection. Although a great deal of effort has been made on characterizing the injection-pulling effects, what seems to be lacking, however, is delivering a system frequency response with respect to injection.

This paper is devoted to a study of injection pulling in an LO. The presented approaches differ from the previous ones by analyzing the PLO under injection in discrete-time and frequency domain, providing accurate and efficient computation of the LO pulling effects. The preliminary publication of this study [14] provided brief theoretical presentations on the proposed frequency-domain analysis and discrete-time-domain calculating method in the prediction of the output spectra of an LO pulled by injection signals. This paper substantially expands [14] to include detailed methodology of discrete-time computation. In addition, a dual-loop model in an expansion of the frequency-domain approach is provided on the phase noise for a pulled LO. An angle-modulated injection signal is also considered to imitate a practical interference from a wireless communication system. 


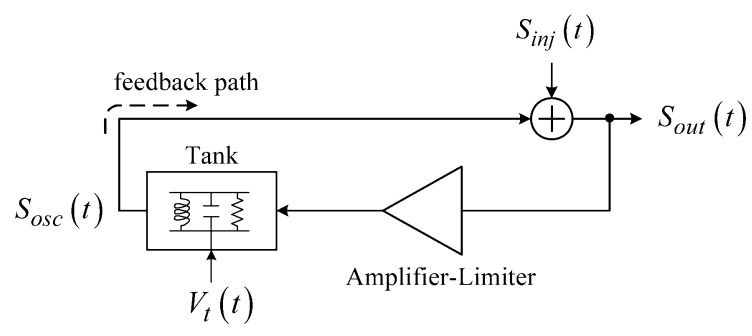

Fig. 2. Block diagram of an oscillator under injection.

This paper is organized as follows. In Section II, we first begin by deriving the locking equation for a VCO under the influence of angle-modulated injection. The remaining content in this section provides a further analysis pertaining to the proposed approach for a PLO under injection. Section III finds the numerical solution of the locking equation in discrete-time domain, which can accurately predict the output spectra distorted due to sinusoidal or angle-modulated injection, to evaluate the spurious performance of a PLO under injection. Section IV presents the frequency-domain PLO model to derive the injection transfer function which, as a result, shows a frequency response characteristic of bandpass type to the spurious tones caused by an injection signal. This section also provides the noise transfer functions to evaluate the overall output phase noise for a PLO under injection. An attractive aspect of introducing the noise transfer functions is their property of shaping the noises with respect to each noise source. Section V discusses the theoretically predicted and experimental results. Finally, Section VI summarizes and concludes this paper.

\section{INJECTION-LOCKING EQUATIONS}

This section derives the locking equation for a VCO under injection. This is the first step toward developing a detailed model of a PLO under the influence of injection. An angle-modulated injection is considered and will be used in the characterization of its influence on a PLO. Fig. 2 displays a simplified VCO model, which consists of an amplifier limiter, a tunable tank circuit, and a feedback path. The amplifier limiter is an amplifier with a nonlinear element to stabilize the amplitude of a self-excited oscillator. The tank circuit has a resonant frequency controlled by the tuning voltage $V_{t}(t)$ to determine the frequency of the VCO. The feedback path forms a loop to establish the oscillation condition. Note that the feedback path includes a summing point to allow injection of signals. In this figure, $S_{\text {osc }}(t)$ represents the inherent VCO output, $S_{\mathrm{inj}}(t)$ represents the injection signal, $S_{\text {out }}(t)$ is the resultant VCO output under injection, and $V_{t}(t)$ is the tuning voltage that controls the inherent VCO frequency.

\section{A. Generalized Locking Equation}

Based on the vector diagram used in Adler's work [3], Fig. 3 illustrates the vector representation of the signals, as shown in Fig. 2. Consider that $S_{\text {inj }}(t)$ has a constant amplitude $E_{\text {inj }}$ and an instantaneous frequency $\omega_{\text {inj }}(t)$ that can be expressed as

$$
\omega_{\text {inj }}(t)=\omega_{\text {inj }}+\frac{d \theta_{i}(t)}{d t}
$$

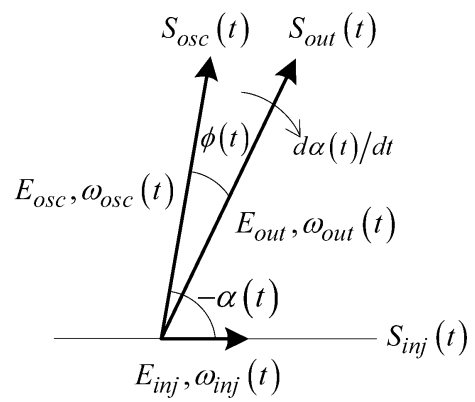

Fig. 3. Vector diagram of the signals shown in Fig. 2.

where $\omega_{\text {inj }}$ is the injection center frequency and $d \theta_{i}(t) / d t$ represents the instantaneous modulation frequency of $S_{\mathrm{inj}}(t)$. When the injection signal is purely sinusoidal, $\theta_{i}(t)$ is a constant and $d \theta_{i}(t) / d t$ is equal to zero. The inherent VCO output $S_{\text {osc }}(t)$ has a constant amplitude $E_{\text {osc }}$ in nature and the VCO is running at frequency $\omega_{\text {osc }}(t)$ determined by the tank circuit. The VCO inherent frequency $\omega_{\text {osc }}(t)$ can be represented as

$$
\omega_{\text {osc }}(t)=\omega_{\text {osc }}+\frac{d \theta_{o}(t)}{d t}
$$

where $\omega_{\text {osc }}$ is the oscillation center frequency and $\theta_{o}(t)$ denotes the phase variation of $S_{\text {osc }}(t)$. When the VCO is phase locked, $\theta_{o}(t)$ can be regarded as a result of phase correction produced by a PLL. The resultant VCO output signal $S_{\text {out }}(t)$ shown in Fig. 3 is also considered to have a constant amplitude $E_{\text {out }}$ and an instantaneous frequency $\omega_{\text {out }}(t)$. In Fig. 3, the phase difference $\phi(t)$ between $S_{\text {osc }}(t)$ and $S_{\text {out }}(t)$ results from the addition of $S_{\mathrm{inj}}(t)$ [3], and $-\alpha(t)$ defines the phase difference between $S_{\text {osc }}(t)$ and $S_{\mathrm{inj}}(t)$. Consider that $S_{\mathrm{inj}}(t)$ is always at rest with respect to our eyes, $S_{\text {out }}(t)$ can be regarded as a vector rotating clockwise with an instantaneous beat frequency $d \alpha(t) / d t$ with respect to $S_{\text {inj }}(t)$. Therefore, $\omega_{\text {out }}(t)$ shall be represented as

$$
\omega_{\text {out }}(t)=\omega_{\text {inj }}(t)+\frac{d \alpha(t)}{d t} .
$$

Unlike Adler's analysis in [3], we proceed with the derivation by considering that the VCO inherent frequency and injection signal frequency has an instantaneous variation $d \theta_{o}(t) / d t$ and $d \theta_{i}(t) / d t$, respectively. Adler's equation in [3] can then be rewritten as

$$
\frac{d \alpha(t)}{d t}=-\frac{\omega_{\mathrm{osc}}(t)}{2 Q} \frac{E_{\mathrm{inj}}}{E_{\mathrm{osc}}} \sin \alpha(t)+\Delta \omega_{\mathrm{osc}}(t)
$$

where

$$
\begin{aligned}
\Delta \omega_{\mathrm{osc}}(t) & =\left[\omega_{\mathrm{osc}}+\frac{d \theta_{o}(t)}{d t}\right]-\left[\omega_{\mathrm{inj}}+\frac{d \theta_{i}(t)}{d t}\right] \\
& =\Delta \omega_{\mathrm{osc}}+\frac{d \theta_{o}(t)}{d t}-\frac{d \theta_{i}(t)}{d t}
\end{aligned}
$$

is the undisturbed beat frequency that defines the spontaneous instantaneous frequency separation between the inherent VCO output and injection signal. Note that $\Delta \omega_{\mathrm{osc}}=\left(\omega_{\mathrm{osc}}-\omega_{\mathrm{inj}}\right)$ defines the frequency separation between inherent oscillation and injection center frequencies. Equation (4) is the generalized 


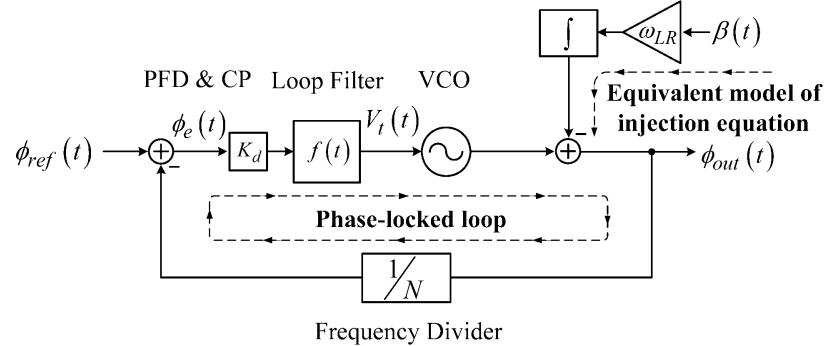

Fig. 4. Time-domain model to account for phase dynamics of a PLO under injection.

locking equation that describes phase dynamics from the timevarying phase and amplitude relationship between a VCO and an angle-modulated injection signal.

\section{B. Injection Equation}

Consider that $S_{\text {osc }}(t)$ is not from a free-running oscillator, but instead from a PLO controlled by a PLL. The PLL phaselocking mechanism can dynamically correct the oscillation frequency via a tuning voltage $V_{t}(t)$, and thus the instantaneous frequency variation $d \theta_{o}(t) / d t$ of the VCO can be replaced by $K_{v} V_{t}(t)$, where $K_{v}$ is the tuning sensitivity of the VCO. Therefore, the VCO inherent frequency in (2) can be further mathematically expressed as

$$
\omega_{\mathrm{osc}}(t)=\omega_{\mathrm{osc}}+K_{v} V_{t}(t) .
$$

Substituting (4) and (6) into (3), the resultant PLO output frequency $\omega_{\text {out }}(t)$ can be found as

$$
\begin{aligned}
\omega_{\text {out }}(t)= & {\left[\omega_{\mathrm{osc}}+K_{v} V_{t}(t)\right] } \\
& {\left[1-\frac{1}{2 Q} \frac{E_{\mathrm{inj}}}{E_{\mathrm{osc}}} \sin \alpha(t)\right] } \\
\approx & \omega_{\mathrm{osc}}+K_{v} V_{t}(t)-\omega_{\mathrm{LR}} \beta(t)
\end{aligned}
$$

where $\omega_{\mathrm{LR}}=\omega_{\mathrm{osc}} E_{\mathrm{inj}} / 2 Q E_{\mathrm{osc}}$ is interpreted as the locking range of a free-running oscillator [3], and $\beta(t)$ is equal to $\sin \alpha(t)$ and is regarded as an equivalent injection-induced source to cause frequency modulation in a PLO. Integrating (7) yields the resultant PLO output phase as

$$
\phi_{\text {out }}(t)=\omega_{\text {osc }} t+K_{v} \int V_{t}(t) d t+\theta_{0}-\omega_{\mathrm{LR}} \int \beta(t) d t
$$

where $\theta_{0}$ is an initial oscillation phase. It is noted that (8) interprets the resultant PLO output phase as a combination of the phase-locking mechanism and the injection-locking process.

Fig. 4 shows a combined model of the PLL and the injection equation (8) to account for phase dynamics of a PLO under injection. In this figure, $K_{d}$ is the combined gain of the PFD and CP, $f(t)$ represents impulse response of the loop filter, and $N$ is the divider modulus. $\phi_{\text {ref }}(t)$ and $\phi_{e}(t)$ express the reference phase and the output phase error of the PFD, respectively. The tuning voltage governed by the phase-locking mechanism in the PLL is

$$
V_{t}(t)=K_{d} \phi_{e}(t) * f(t)
$$

where $*$ denotes the convolution operator, and

$$
\phi_{e}(t)=\phi_{\text {ref }}(t)-\frac{\phi_{\text {out }}(t)}{N} .
$$

\section{DISCRETE-TIME-DOMAIN APPROACH}

For a PLO under injection, the injection-locking process generally interacts with the PLL phase-locking mechanism. This indicates that $V_{t}(t)$ and $\beta(t)$ in (8) are mutually dependent. As a general means toward calculating the output spectrum of a PLO under injection, this paper proposes a discrete-time-domain approach [15] to solve the problem. To begin with, we first examine the locking equation (4) derived in Section II. Making a discretization of (4) obtains

$$
(\alpha[n]-\alpha[n-1]) / T=-\frac{\omega_{\mathrm{osc}}[n]}{2 Q} \frac{E_{\mathrm{inj}}}{E_{\mathrm{osc}}} \beta[n]+\Delta \omega_{\mathrm{osc}}[n]
$$

where $n$ is the index of data sequences, a time interval $T=$ $\left(t_{n}-t_{n-1}\right)$ is the calculation time step, and $t_{n}$ represents the time at which the $n$th sampling occurs. Making a rearrangement of (11) yields

$$
\alpha[n]=\left(-\frac{\omega_{\mathrm{osc}}[n]}{2 Q} \frac{E_{\mathrm{inj}}}{E_{\mathrm{osc}}} \beta[n]+\Delta \omega_{\mathrm{osc}}[n]\right) T+\alpha[n-1]
$$

where

$$
\begin{aligned}
\Delta \omega_{\mathrm{osc}}[n] & =\omega_{\mathrm{osc}}[n]-\omega_{\mathrm{inj}}[n] \\
& =\Delta \omega_{\mathrm{osc}}+K_{v} V_{t}[n]-\left(\theta_{i}[n]-\theta_{i}[n-1]\right) / T .
\end{aligned}
$$

In (12), $\beta[n]$ is approximated as $\sin \alpha[n-1]$ under the assumption that $T$ is small. Empirically, this assumption is satisfied for $T$ given as a fraction of the inverse of the frequency separation between inherent oscillation and injection center frequencies. In the application examples of this paper, such a frequency separation ranges from several tens of kilohertz to several megahertz, and therefore, $T$ is set from a few hundredths to several microsecond in the discrete-time-domain calculations. It is noted that once this frequency separation is smaller than the PLL reference frequency, we should use the PLL reference frequency instead to determine $T$ for ensuring the accuracy in the PLL response calculations. With the earlier approximation, $\alpha[n]$ in (12) can be recursively calculated instead of solving it at time instant $t_{n}$.

Consider that the output signal for a PLO under injection is given by

$$
S_{\text {out }}(t)=\operatorname{Re}\left\{E_{\text {out }} e^{j\left[\omega_{\text {osc }} t+\phi_{m}(t)\right]}\right\}
$$

where $E_{\text {out }}$ denotes the oscillator amplitude and $\phi_{m}(t)$ is regarded as an oscillator phase modulation function caused by the 
interaction between PLL and injection-locking processes. The following calculation to determine the output signal only involves the oscillator phase modulation function $\phi_{m}(t)$ without considering the carrier frequency $\omega_{\text {osc }}$, and therefore, has the advantages of a fast computation time. From (8), $\phi_{m}(t)$ can be found as

$$
\phi_{m}(t)=K_{v} \int V_{t}(t) d t+\theta_{0}-\omega_{\mathrm{LR}} \int \beta(t) d t .
$$

In the discrete-time domain, this oscillator phase modulation function can be represented as

$$
\phi_{m}[n]=K_{v} V_{t}[n] n T+\theta_{0}+\phi_{\alpha}[n]
$$

where

$$
\phi_{\alpha}[n]=\phi_{\alpha}[n-1]-\omega_{\mathrm{LR}} \beta[n] T
$$

With the discrete-time representations of (9) and (10), $V_{t}[n]$ in (16) can be shown as

$$
V_{t}[n]=K_{d} \phi_{e}[n] * f[n]
$$

where

$$
\phi_{e}[n]=\phi_{0}-\phi_{m}[n-1] / N
$$

and $f[n]$ is the discrete-time impulse response of the loop filter. It is noted that $\phi_{0}$ in (19) is a constant reference phase.

To obtain the RF output signal, the phase modulation function is finally expressed as a pulse train weighted by $\phi_{m}[n]$ expressed in (16). The VCO output waveform can be found using the form [15]

$$
S_{\text {out }}(t)=E_{\text {out }} \cos \left(\omega_{\text {osc }} t+\sum_{n} \phi_{m}[n] h_{r}(t-n T)\right)
$$

where $h_{r}(t)$ is the impulse response of the reconstruction filter. The VCO output spectrum can then be predicted by taking the Fourier transform of (20).

\section{FREQUENCY-DOMAIN APPROACH}

Section III derives the governing equations of the PLO output phase in time domain under the influence of injection. In this section, we begin by exploring the PLO output phase in frequency domain when an injection signal is applied. The benefit of the frequency-domain approach is the comprehensibility of frequency response with respect to the signals of interest. Fig. 5 displays the counterpart of the PLO model under injection in frequency domain. In this figure, $F(s)$ represents the transfer function of the loop filter; $\phi_{\text {ref }}(s)$ and $\phi_{e}(s)$ express the input reference source and the output phase error of the PFD, respectively. $\beta(s)$ and $\phi_{\text {out }}(s)$ is the Laplace transform of $\beta(t)$ and $\phi_{\text {out }}(t)$, respectively.

Based on the frequency-domain approach, the phase noise of an injection-locked oscillator (ILO) is first examined. The phase noise of a PLO under injection is then explored. We will focus

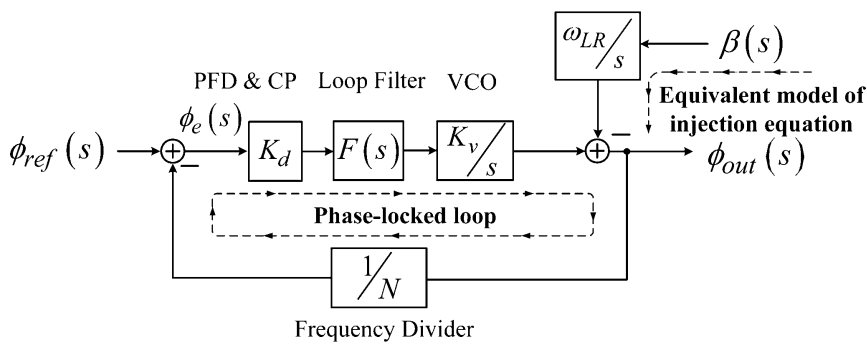

Fig. 5. Frequency-domain model to account for phase dynamics of a PLO under injection.

particularly on the dependence of the PLO output phase noise on the injection phase noise in relation to noise transfer functions when the injection frequency is identical to the synthesized frequency.

\section{A. Injection Transfer Function}

In PLL analysis, the prevalent method is to use the forwardpath transfer function $G(s)$ and feedback-path transfer function $H(s)$ to formulate the Laplace-domain loop equations. The value of introducing $G(s)$ and $H(s)$ is that all the transfer functions of interest within the PLL can be parameterized by them and can be easily related to one another. As illustrated in Fig. 5, the output phase can be found as

$$
\phi_{\text {out }}(s)=H_{c}(s) \phi_{\text {ref }}(s)-H_{\text {inj }}(s) \beta(s)
$$

where

$$
H_{c}(s)=\frac{G(s)}{1+G(s) H(s)}
$$

represents the closed-loop transfer function that behaves like a low-pass filter, and

$$
H_{\mathrm{inj}}(s)=\frac{\omega_{\mathrm{LR}}}{s[1+G(s) H(s)]}
$$

is the transfer function with respect to the injection that behaves like a bandpass filter. It is noted that the forward- and feedback-path transfer functions of the PLL in Fig. 5 are $G(s)=$ $K_{d} F(s) K_{v} / s$ and $H(s)=1 / N$.

Assuming that a second-order loop is considered, (23) can be further expressed as

$$
H_{\mathrm{inj}}(s)=\frac{\omega_{\mathrm{LR}} s}{s^{2}+2 \varsigma \omega_{n} s+\omega_{n}^{2}}
$$

parameterized by the natural frequency $\omega_{n}$ and the damping factor $\varsigma$ [16]. Taking the absolute value of (24) with $s=j \omega$, we obtain

$$
\left|H_{\text {inj }}(s=j \omega)\right|=\frac{\omega_{\mathrm{LR}} \omega}{\sqrt{4 \varsigma^{2} \omega_{n}^{2} \omega^{2}+\left(\omega^{2}-\omega_{n}^{2}\right)^{2}}}
$$

where $\omega$ is the frequency offset from the synthesized frequency. It is particularly noted that in [10], Razavi has derived the same expression as (25) using a time-domain approach. However, this frequency-domain approach based on the widely used PLL 
transfer functions is more straightforward to understand. By setting the derivative of (25) equal to zero, the injection transfer function is found to have a peak magnitude occurring at $\omega_{n}$, i.e.,

$$
G_{p}=\left|H_{\mathrm{inj}}\left(s=j \omega_{n}\right)\right|=\frac{\omega_{\mathrm{LR}}}{2 \varsigma \omega_{n}}=\frac{\omega_{\mathrm{osc}} E_{\mathrm{inj}}}{4 Q \varsigma \omega_{n} E_{\mathrm{osc}}} .
$$

It is indicated from (26) that a VCO with a high quality factor, as well as a PLL with high natural frequency and a high damping factor can help reduce the injection-pulling effects.

\section{B. Noise Transfer Functions of an ILO}

Over the last few decades, the phase noise of a synchronized oscillator using injection locking has been reported by many authors [17]-[22]. Differing from the earlier studies, this paper is devoted to a phase noise model by using the generalized locking equation shown as (4) to establish an equivalent injection-locked loop for an ILO. To begin with, we first consider that the oscillator is locked by a sinusoidal injection signal and reaches its steady state. In the steady state, the phase difference $\alpha(t)$ between $S_{\mathrm{inj}}(t)$ and $S_{\mathrm{osc}}(t)$ approaches a constant value, which can be denoted as $\alpha_{\infty}$. Next, we form an approximation of (4) based on the assumption that the inherent oscillator output and injection signal are sinusoidal and the injection level is weak so that $\alpha_{\infty}$ can be solved with the following locking equation under locking condition:

$$
\frac{d \alpha_{\infty}}{d t}=-\omega_{\mathrm{LR}} \sin \alpha_{\infty}+\Delta \omega_{\mathrm{osc}}=0 .
$$

Let us turn to a consideration that the injection frequency and the inherent oscillation frequency are disturbed by phase noise $\phi_{n, i}(t)$ and $\phi_{n, 0}(t)$, i.e., $\omega_{\text {inj }}(t)=\omega_{\text {inj }}+d \phi_{n, i}(t) / d t$ and $\omega_{\text {osc }}(t)=\omega_{\text {osc }}+d \phi_{n, 0}(t) / d t$. Assume that the phase difference between $S_{\mathrm{inj}}(t)$ and $S_{\mathrm{osc}}(t)$ can be represented as $\alpha_{\mathrm{ss}}=$ $\left(\alpha_{\infty}+\alpha_{n}\right)$ with a phase perturbation $\alpha_{n}$ induced by the phase disturbance of $\phi_{n, i}(t)$ and $\phi_{n, 0}(t)$. In the steady state, substituting into (4) gives

$$
\frac{d \alpha_{\mathrm{ss}}}{d t}=-\omega_{\mathrm{LR}} \sin \alpha_{\mathrm{ss}}+\Delta \omega_{\mathrm{osc}}+\frac{d \phi_{n, 0}(t)}{d t}-\frac{d \phi_{n, i}(t)}{d t} .
$$

Making the Taylor's expansion of $\sin \alpha_{\mathrm{ss}}$ around $\alpha_{\infty}$ gives

$$
\sin \alpha_{\mathrm{ss}} \approx \sin \alpha_{\infty}+\alpha_{n} \cos \alpha_{\infty}
$$

Substituting into (28) with the locking condition (27) leads to

$$
\frac{d \alpha_{n}}{d t}=-\omega_{\mathrm{LR}} \alpha_{n} \cos \alpha_{\infty}+\frac{d \phi_{n, 0}(t)}{d t}-\frac{d \phi_{n, i}(t)}{d t} .
$$

The resultant output frequency can then be found as

$$
\omega_{\text {out }}(t)=\omega_{\text {inj }}-\omega_{\text {LR }} \alpha_{n} \cos \alpha_{\infty}+\frac{d \phi_{n, 0}(t)}{d t} .
$$

Integrating (31) gives the phase perturbation $\phi_{n, \text { out }}(t)$ around the ILO frequency $\omega_{\text {inj }}$, i.e.,

$$
\phi_{n, \text { out }}(t)=-\omega_{\mathrm{LR}} \cos \alpha_{\infty} \int \alpha_{n} d t+\phi_{n, 0}(t) .
$$

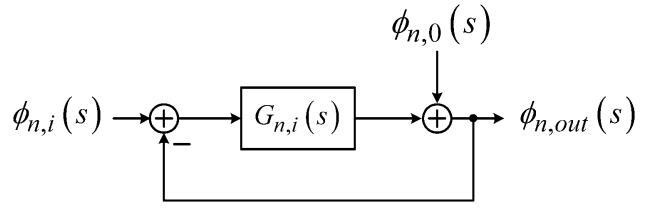

Fig. 6. Injection-locked loop for phase-noise analysis.

Continuing with the derivation, write the aforementioned equations with their frequency-domain representations $\alpha_{n}(s), \phi_{n, i}(s)$ and $\phi_{n, 0}(s)$, making a series of substitutions and rearrangements yields

$$
\phi_{n, \text { out }}(s)=H_{n, 0}(s) \phi_{n, 0}(s)+H_{n, i}(s) \phi_{n, i}(s)
$$

where

$$
H_{n, 0}(s)=\frac{s}{\left(s+\omega_{\mathrm{LR}} \cos \alpha_{\infty}\right)}
$$

shows a property of high-pass filtering with respect to $\phi_{n, 0}(s)$, and

$$
H_{n, i}(s)=\frac{\omega_{\mathrm{LR}} \cos \alpha_{\infty}}{\left(s+\omega_{\mathrm{LR}} \cos \alpha_{\infty}\right)}
$$

shows a property of low-pass filtering with respect to $\phi_{n, i}(s)$. An interpretation of phase noise associated with such an approach reveals that the overall phase noise can be regarded as the combination of a high-pass filtered inherent VCO phase noise and a low-pass filtered injection phase noise. According to (33)-(35), the equivalent injection-locked loop can be represented as Fig. 6 with $G_{n, i}(s)=\omega_{\mathrm{LR}} \cos \alpha_{\infty} / s$. It is noted that the injection-locking process is a feedback system and acts like a first-order PLL.

The VCO phase noise $\phi_{n, 0}(s)$ is assumed to have a power spectral density $S_{n, 0}(\omega)$. The injection phase noise $\phi_{n, i}(s)$ has a power spectral density $S_{n, i}(\omega)$. The power spectral density of the overall phase noise $S_{n \text {,out }}(\omega)$ can then be represented as

$$
S_{n, \text { out }}(\omega)=\left|H_{n, 0}(j \omega)\right|^{2} S_{n, 0}(\omega)+\left|H_{n, i}(j \omega)\right|^{2} S_{n, i}(\omega)
$$

where

$$
\begin{aligned}
\left|H_{n, 0}(j \omega)\right|^{2} & =\frac{\left(\omega / \omega_{\mathrm{LR}}\right)^{2}}{1-\left(\Delta \omega_{\mathrm{osc}} / \omega_{\mathrm{LR}}\right)^{2}+\left(\omega / \omega_{\mathrm{LR}}\right)^{2}} \\
\left|H_{n, i}(j \omega)\right|^{2} & =\frac{1-\left(\Delta \omega_{\mathrm{osc}} / \omega_{\mathrm{LR}}\right)^{2}}{1-\left(\Delta \omega_{\mathrm{osc}} / \omega_{\mathrm{LR}}\right)^{2}+\left(\omega / \omega_{\mathrm{LR}}\right)^{2}}
\end{aligned}
$$

are found by using (34), (35), and the locking condition (27). As seen by (37) and (38), $\omega_{\mathrm{LR}}$ is in a manifest manner on the influence of the overall phase noise. It is noted that Sugiura and Sugimoto derived a similar result to the earlier by using a different approach in [19]. When the injection frequency is identical to the VCO inherent frequency, substituting $\Delta \omega_{\text {osc }}$ with zero yields the same results as reported in [7] and [18]. 


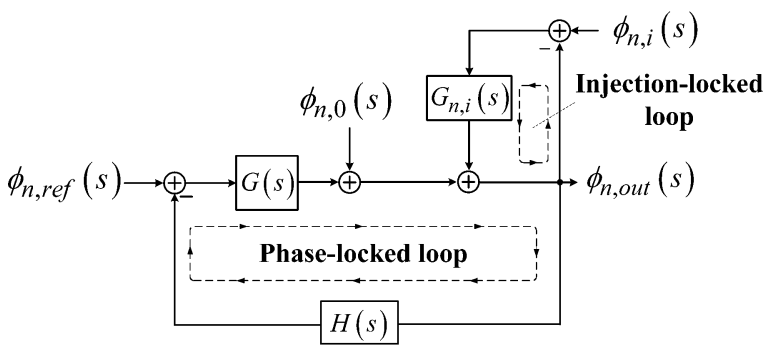

Fig. 7. Dual-loop model for phase noise analysis of a PLO under injection.

\section{Noise Transfer Functions of a PLO}

As previously mentioned, the PLO may be interfered by the power amplifier output signal, which has a center frequency equal to the synthesized frequency. Therefore, the PLO output phase noise in such a situation can be analyzed with $\Delta \omega_{\text {osc }}=0$. Recall the model depicted in Fig. 6, incorporating the PLL mechanism recasts the model to a dual-loop configuration, as shown in Fig. 7 , where $\phi_{n, \text { ref }}(s), \phi_{n, 0}(s), \phi_{n, i}(s)$, and $\phi_{n, \text { out }}(s)$ represents the phase noise of the reference, VCO, injection, and output signal, respectively. The dual-loop model is apparently a combination of the PLL and injection-locked loop, and the overall phase noise $\phi_{n, \text { out }}(s)$ can be formulated as

$$
\begin{aligned}
\phi_{\text {out }, n}(s)=H_{n, \text { ref }}(s) \phi_{n, \text { ref }}(s)+ & H_{n, \text { osc }}(s) \phi_{n, 0}(s) \\
& +H_{n, \text { inj }}(s) \phi_{n, i}(s)
\end{aligned}
$$

where

$$
\begin{aligned}
H_{n, \text { ref }}(s) & =\frac{G(s) H_{n, 0}(s)}{1+G(s) H(s) H_{n, 0}(s)} \\
H_{n, \text { osc }}(s) & =\frac{H_{n, 0}(s)}{1+G(s) H(s) H_{n, 0}(s)} \\
H_{n, \text { inj }}(s) & =\frac{H_{n, i}(s)}{1+G(s) H(s) H_{n, 0}(s)}
\end{aligned}
$$

with $H_{n, 0}(s)$ and $H_{n, i}(s)$ given in (34) and (35), while $\cos \alpha_{\infty}$ is equal to unity, and because of that $\alpha_{\infty}$ is equal to zero in the steady state with $\Delta \omega_{\text {osc }}=0$ [5]. In Fig. 7, the forwardpath transfer function $G_{n, i}(s)$ of the injection-locked loop is equal to $\omega_{\mathrm{LR}} / s$. Equation (39) indicates that the overall phase noise is broken down into its three major components, which are the reference, $\mathrm{VCO}$, and injection phase noise. The noise transfer functions (40)-(42) are obviously in an interaction of the PLL transfer functions $G(s), H(s)$, and the injection-locked loop noise transfer functions $H_{n, 0}(s)$ and $H_{n, i}(s)$. Consider that the reference phase noise $\phi_{n \text {,ref }}(s)$ has a power spectral density $S_{n, \text { ref }}(\omega)$. The power spectral density of the overall phase noise $S_{n \text {,out }}(\omega)$ can then be represented as

$$
\begin{array}{r}
S_{n, \text { out }}(\omega)=\left|H_{n, \text { ref }}(j \omega)\right|^{2} S_{n, \text { ref }}(\omega)+\left|H_{n, \text { osc }}(j \omega)\right|^{2} S_{n, 0}(\omega) \\
+\left|H_{n, \text { inj }}(j \omega)\right|^{2} S_{n, i}(\omega) .
\end{array}
$$

The overall phase noise of a PLO under injection can be predicted by using (43).

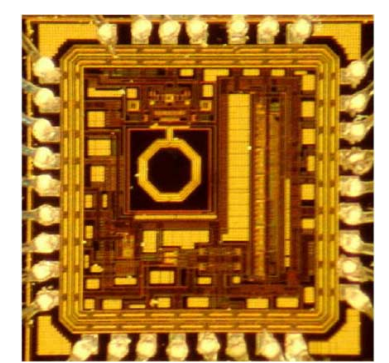

Fig. 8. Die photograph of the implemented CMOS PLL frequency synthesizer.

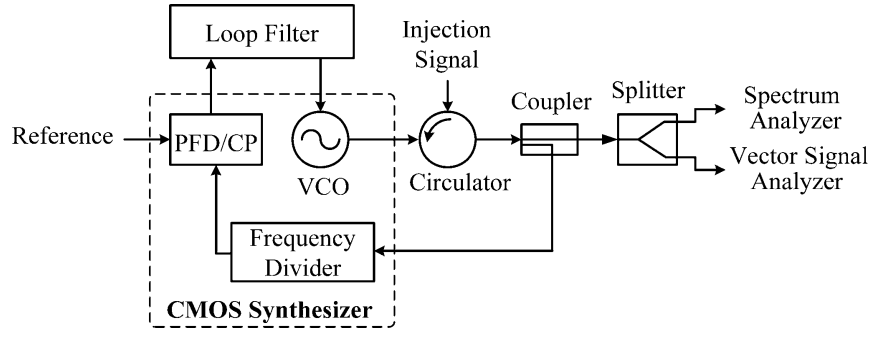

Fig. 9. PLO test setup for injection experiments.

\section{Measured and Calculated Results}

An $S$-band PLL frequency synthesizer has been designed and implemented in $0.18-\mu \mathrm{m}$ CMOS process for this study. The die photograph is shown in Fig. 8. This chip mainly contains a PFD, CP, frequency divider, and VCO. When this synthesizer is locked at $2.46 \mathrm{GHz}$, it delivers an output power of $1.44 \mathrm{dBm}$ with an estimated natural frequency and damping factor of $63.23 \mathrm{kHz}$ and 1.54, respectively. By referring to [5], the setup for testing the synthesizer is depicted in Fig. 9, which indicates that an injection signal is fed to the $\mathrm{VCO}$ output port and simultaneously isolated from the VCO output signal with the help of a circulator.

The following sections demonstrate the measured and calculated results of the PLO influenced by different injection conditions. First, a sinusoidal signal is used as an injection signal of the PLO to validate the proposed discrete-time- and frequency-domain approaches for predicting the output spectrum and phase noise. Second, an injection signal of Gaussian minimum-shift keying (GMSK) modulation is applied to the PLO as a scenario for studying the LO pulling effects. With the help of the proposed discrete-time calculating method, the predicted spectrum is provided to account for the spurious performance of a pulled LO. In addition, the phase noise is also estimated using the theoretical result from (43).

\section{A. Sinusoidal Signal Injection}

For clarity, we first define a few symbols that will be used in the following discussions.

$$
\begin{aligned}
& f_{0}=2.46 \mathrm{GHz}=\text { synthesized frequency of PLL. } \\
& f_{\mathrm{inj}}=\text { frequency of injection signal. } \\
& \Delta f=\left|f_{0}-f_{\text {inj }}\right|=\text { injection frequency offset from } f_{0} . \\
& P_{\text {inj }}=\text { power level of injection signal. }
\end{aligned}
$$

The experiment first applies a sinusoidal injection signal with frequency $f_{\text {inj }}$ to the PLO. $f_{\text {inj }}$ is chosen with different 


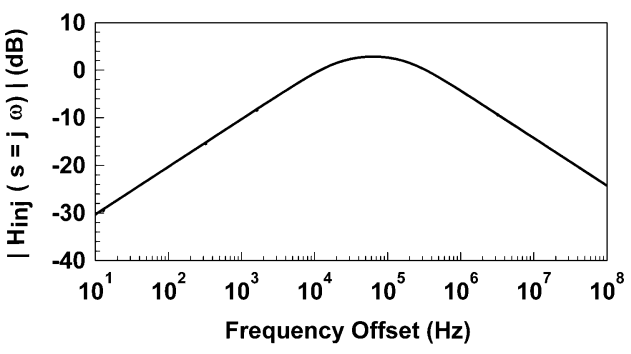

Fig. 10. Magnitude response of the injection transfer function for the PLL frequency synthesizer locked at $2.46 \mathrm{GHz}$.

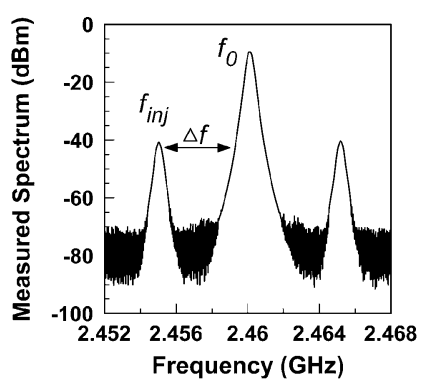

(a)

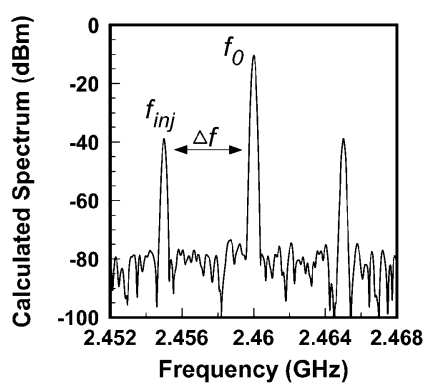

(b)
Fig. 11. Output spectrum of the PLO with a sinusoidal injection signal having a frequency offset of $5 \mathrm{MHz}$. (a) Measured results. (b) Calculated results.

values departing from the synthesized frequency $f_{0}$ to observe the change of spurious outputs. Second, a sinusoidal injection applies to the PLO with $f_{\text {inj }}$ identical to $f_{0}$ at various injection power levels $P_{\text {inj }}$ in consideration of observing the phase noise.

In this experiment, $P_{\text {inj }}$ is set at $40 \mathrm{~dB}$ below the $1.44-\mathrm{dBm}$ oscillator output power. Fig. 10 plots the magnitude response of the injection transfer function (25). As mentioned, it shows a bandpass-type response with a peak magnitude of $2.85 \mathrm{~dB}$ at the frequency offset $\Delta f$ equal to the natural frequency $(63.23 \mathrm{kHz})$. When $\Delta f$ is chosen as $5 \mathrm{MHz}$ and 500 and $20 \mathrm{kHz}$, the corresponding injection transfer function magnitudes calculated are $-11.25,-1.49$, and $1.51 \mathrm{~dB}$, respectively. These calculated magnitudes are related to the level of the spurious tones caused by the injection signal.

Figs. 11-13 compare the theoretically predicted output spectra using the proposed discrete-time calculating method with the measured ones for these three cases of different $\Delta f$. The comparisons show very good agreement. The PLO under the sinusoidal injection generates a number of spurious tones with tone spacing equal to $\Delta f$. It can be observed from Figs. 11-13 that the maximum two spurious tone levels occur at the frequencies $f_{0} \pm \Delta f$. For the case of $\Delta f=5 \mathrm{MHz}$, the PLO output spectrum shown in Fig. 11 exhibits similar spurious tone levels at $f_{0} \pm \Delta f$, and both tone levels are much less than the synthesized one at $f_{0}$. The aforementioned characteristics are classified as weak injection-pulling effects. As shown in Figs. 12 and 13, the injection-pulling effects become stronger as $\Delta f$ approaches to $63.23 \mathrm{kHz}$, the natural frequency of the PLL. One can see from Figs. 12 and 13 that the two spurious tone levels at $f_{0} \pm \Delta f$ are not symmetric with respect to $f_{0}$, and both tone levels grow to approach the synthesized one. In Fig. 13, the maximum spurious tone level at $f_{\text {inj }}$ even exceeds the synthesized one at $f_{0}$. This corresponds to the positive

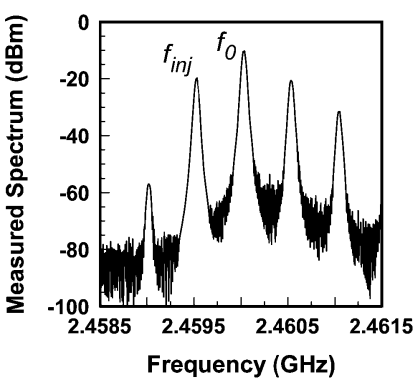

(a)

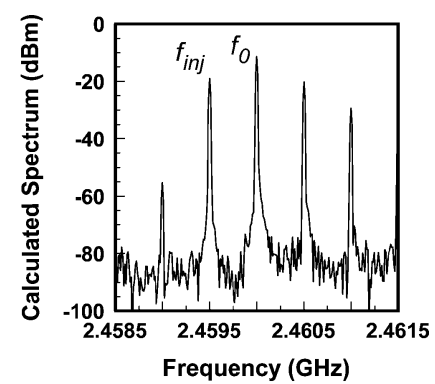

(b)
Fig. 12. Output spectrum of the PLO with a sinusoidal injection signal having a frequency offset of $500 \mathrm{kHz}$. (a) Measured results. (b) Calculated results.

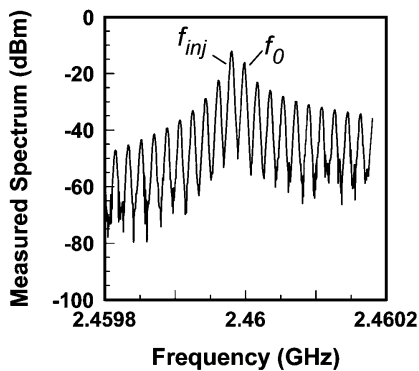

(a)

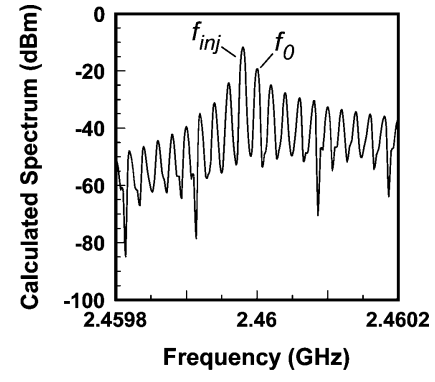

(b)
Fig. 13. Output spectrum of the PLO with a sinusoidal injection signal having a frequency offset of $20 \mathrm{kHz}$. (a) Measured results. (b) Calculated results.

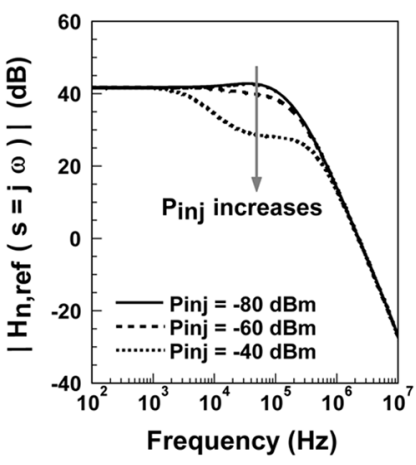

(a)

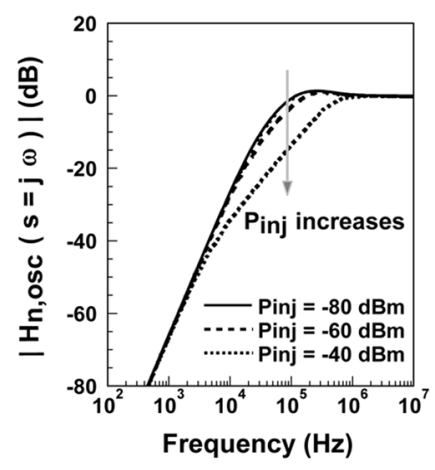

(b)
Fig. 14. Magnitude response of the noise transfer functions at various injection power levels. (a) Reference noise transfer function. (b) Oscillation noise transfer function.

magnitude of the injection transfer function in Fig. 10 at a frequency offset of $20 \mathrm{kHz}$. It is noted that the computation time required for individual output spectrum calculations shown in Figs. 11-13 is less than $90 \mathrm{~s}$ on a general personal computer.

Another condition was performed with a sinusoidal injection, which has a frequency $f_{\text {inj }}$ identical to the synthesized frequency $f_{0}$, i.e., $\Delta f=0$. The injection power levels are chosen as $-80,-60$, and $-40 \mathrm{dBm}$, which correspond to locking ranges of 3.2, 32, and $320 \mathrm{kHz}$, respectively, of the oscillator. Figs. 14 and 15 plot the magnitude of noise transfer functions derived in (40)-(42). Fig. 14(a) shows the magnitude response of the reference noise transfer function while the solid line, broken line, and dotted line express the response with $P_{\text {inj }}$ equal to $-80,-60$, and $-40 \mathrm{dBm}$, respectively. When $P_{\text {inj }}$ increases, 


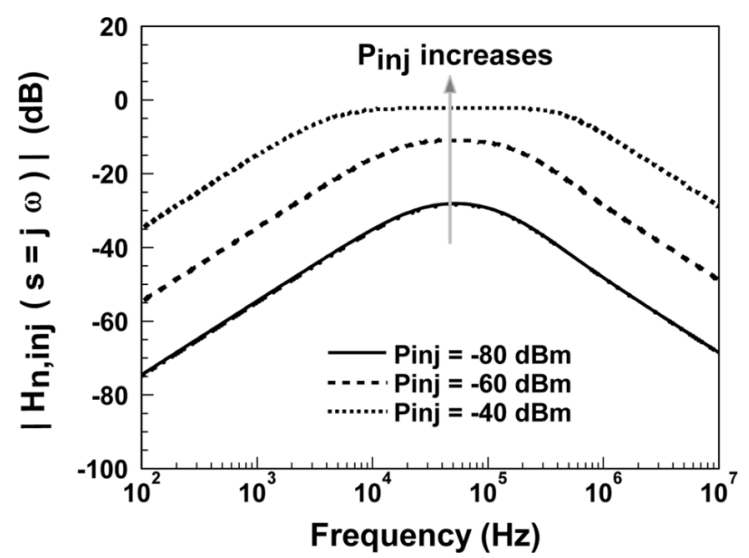

Fig. 15. Magnitude response of the injection noise transfer function at various injection power levels.

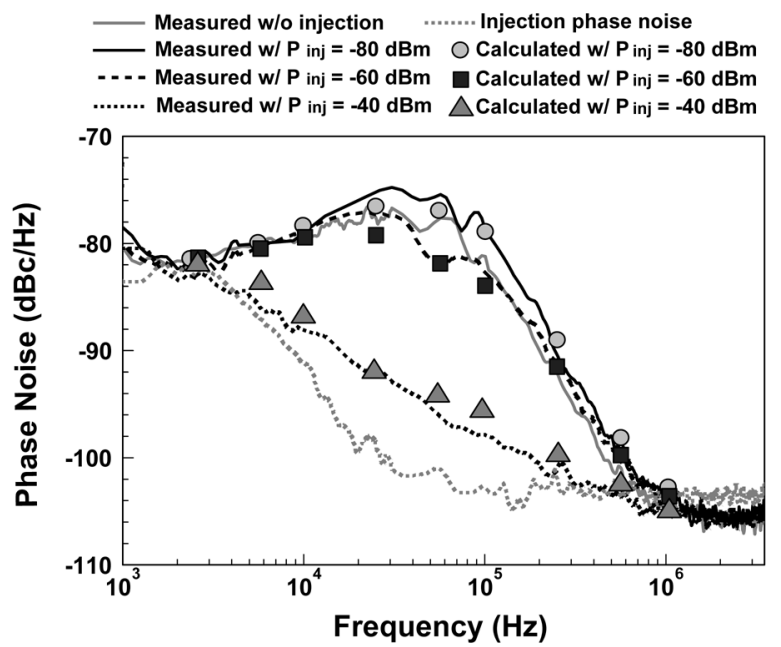

Fig. 16. Comparison of phase noise between measured and calculated results for the PLO with a sinusoidal injection signal having no frequency offset.

the magnitude goes lower around the natural frequency; it reveals that the reference phase noise is further suppressed when $P_{\text {inj }}$ increases. Fig. 14(b) displays the magnitude response of the oscillation noise transfer function. Similarly, it also reveals that the VCO phase noise is further suppressed when $P_{\text {inj }}$ increases. Fig. 15 exhibits the magnitude response of the injection noise transfer function. The magnitude goes higher around the natural frequency when $P_{\text {inj }}$ increases; it indicates that the injection phase noise rises when $P_{\text {inj }}$ increases. To sum up in a word, the overall phase noise will be dominated by the injection phase noise when $P_{\text {inj }}$ continues to increase, since reference and $\mathrm{VCO}$ phase noises are suppressed while injection phase noise is flourishing.

Fig. 16 shows the measured and calculated phase noises at various injection power levels. The measured results are drawn with lines, while the calculated results are drawn with symbols. The solid line in gray is the measured PLO output phase noise without injection. The dotted line in gray is the measured sinusoidal injection signal phase noise. The solid line, broken line, and dotted line in black express the measured phase noise of the PLO output with $P_{\text {inj }}$ equal to $-80,-60$, and $-40 \mathrm{dBm}$, which

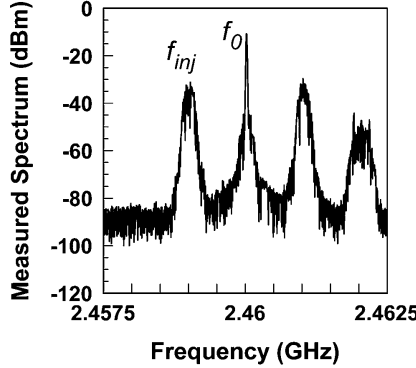

(a)

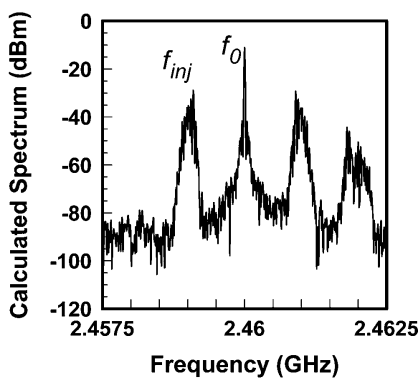

(b)
Fig. 17. Output spectrum of the PLO with a GMSK injection signal having a frequency offset of $1 \mathrm{MHz}$. (a) Measured results. (b) Calculated results.

correspond to locking ranges of $3.2,32$, and $320 \mathrm{kHz}$ of the oscillator, respectively. The circle, square, and triangle symbols express the calculated phase noise of the PLO output by (43) with $P_{\text {inj }}$ equal to $-80,-60$, and $-40 \mathrm{dBm}$, respectively. The comparisons show very good agreement. Consider the condition that $P_{\text {inj }}$ is equal to $-80 \mathrm{dBm}$, the overall phase noise slightly rises. This is because that the suppression on reference and VCO phase noises is insignificant with such a weak injection level. When $P_{\text {inj }}$ increases from -80 to $-40 \mathrm{dBm}$, the overall phase noise significantly goes lower around the natural frequency as mentioned.

\section{B. Modulated Signal Injection}

This experiment used a GMSK signal with a $270.833-\mathrm{kb} / \mathrm{s}$ data rate as an injection signal to imitate interference from the global system for mobile communications (GSM). In this experiment, the injection frequency offset $\Delta f$ is set at $1 \mathrm{MHz}$ from $f_{0}$. Fig. 17 compares the theoretically predicted output spectrum with the measured one for the PLO under such a modulated injection. The comparison again shows excellent agreement. Since the injection frequency offset is large for this case, the characteristics of the weak injection-pulling effects including symmetric and low-spurious phase modulation products at the injection frequency and image injection frequency are observed in Fig. 17. It is also noted that the computation time required for the output spectrum calculation shown in Fig. 17 is about $120 \mathrm{~s}$.

Another condition was also performed with an injection of GMSK signal, which has a center frequency $f_{\text {inj }}$ identical to the synthesized frequency $f_{0}$, i.e., $\Delta f=0$. This imitates the interference originating from parasitic coupling of the PA output in a direct-conversion GSM transmitter. The injection power levels are chosen as $-80,-60$, and $-40 \mathrm{dBm}$, which correspond to locking ranges of $3.2,32$, and $320 \mathrm{kHz}$, respectively, of the oscillator. Fig. 18 shows an example of PLO output spectra under the condition that $P_{\text {inj }}$ is equal to $-60 \mathrm{dBm}$. Fig. 18(a) is the measured results and Fig. 18(b) is the calculated results, both of them show a severe degradation in spectral purity. Fig. 19 compares the measured and calculated phase noises at various injection power levels. The measured results are drawn with lines, while the calculated results are drawn with symbols. The solid line in gray is the measured PLO output phase noise without injection. The solid line, broken line, and dotted line in black express the measured phase noise of the PLO output with $P_{\text {inj }}$ 


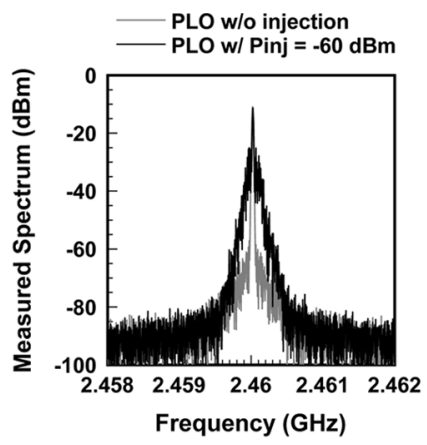

(a)

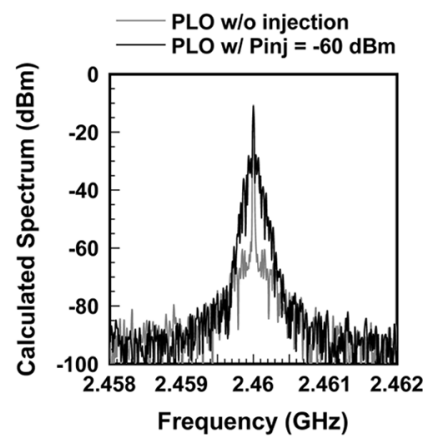

(b)
Fig. 18. Output spectrum of the PLO with a GMSK injection signal having no frequency offset. (a) Measured results. (b) Calculated results.

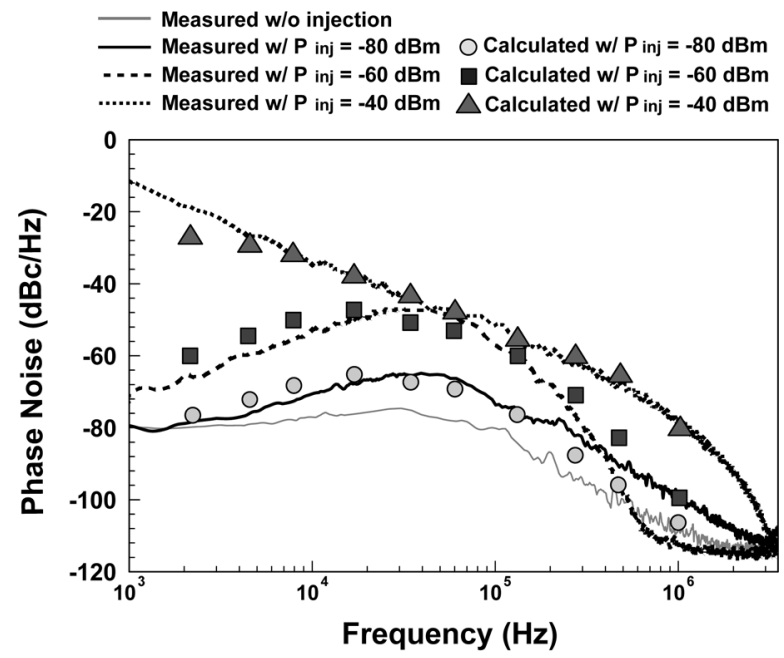

Fig. 19. Comparison of phase noise between measured and calculated results for the PLO with a GMSK injection signal having no frequency offset.

equal to $-80,-60$, and $-40 \mathrm{dBm}$, respectively. The circle, square, and triangle symbols express the calculated phase noise of the PLO output by (43) with $P_{\text {inj }}$ equal to $-80,-60$, and $-40 \mathrm{dBm}$, respectively. The comparisons again show very good agreement. Differing from the results of the sinusoidal injection, the overall phase noise degrades when $P_{\text {inj }}$ increases. This is because the modulated injection signal has a dramatic phase variation, which can be regarded as a phase noise source for the PLO.

It is finally remarked that GSM transmitters suffer from significant performance degradation due to the presence of the LO pulling effects. The impacts include an error vector magnitude increase and a spectral regrowth. Therefore, future work will be dedicated to the deterioration estimation of the transmit signal quality based on the predicted output spectrum and phase noise of a pulled LO in this paper.

\section{CONCLUSION}

This paper has rigorously analyzed the LO pulling effects in discrete time and frequency domain. In the discrete-time domain, the output spectrum of a PLO under injection can be computed in less than $2 \mathrm{~min}$. The calculated output spectra for a
PLO under sinusoidal and modulated injections have been successfully validated by actual measurements. In the frequency domain, an injection transfer function is derived to evaluate the frequency dependence of the spurious outputs caused by the injection signal. In addition, a dual-loop model is developed to account for the phase noise performance of a PLO with a cofrequency injection signal. The calculated and measured results are in good agreement. To conclude, the discrete-time calculating method helps in predicting the output spectrum of a pulled LO and has the capability of co-simulating with other radio frequency components. The frequency-domain approach provides an intuitive and precise characterization of phase noise for an LO interfered by a cochannel signal.

\section{ACKNOWLEDGMENT}

The authors would like to thank the National Chip Implementation Center, Hsinchu, Taiwan, for providing the CMOS foundry service for this paper.

\section{REFERENCES}

[1] B. Razavi, "Challenges in portable RF transceiver design," IEEE Circuits Devices Mag., vol. 12, no. 5, pp. 12-25, Sep. 1996.

[2] B. Razavi, "RF transmitter architectures and circuits," in Proc. IEEE Custom Integr. Circuits Conf., San Diego, CA, 1999, pp. 197-204.

[3] R. Adler, "A study of locking phenomena in oscillators," Proc. IRE, vol. 34, no. 6, pp. 351-357, Jun. 1946.

[4] R. D. Huntoon and A. Weiss, "Synchronization of oscillators," Proc. IRE, vol. 35, no. 12, pp. 1415-1423, Dec. 1947.

[5] R. C. Mackey, "Injection locking of Klystron oscillators," IRE Trans. Microw. Theory Tech., vol. MTT-10, no. 4, pp. 228-235, Jul. 1962.

[6] L. J. Paciorek, "Injection locking of oscillators," Proc. IEEE, vol. 53, no. 11, pp. 1723-1727, Nov. 1965.

[7] K. Kurokawa, "Injection locking of microwave solid-state oscillators," Proc. IEEE, vol. 61, no. 10, pp. 1386-1410, Oct. 1973.

[8] A. Mirzaei, M. E. Heidari, and A. A. Abidi, "Analysis of oscillators locked by large injection signals: Generalized Adler's equation and geometrical interpretation," in Proc. IEEE Custom Integr. Circuits Conf., San Jose, CA, 2006, pp. 737-740.

[9] M. E. Heidari and A. A. Abidi, "Behavioral models of frequency pulling in oscillators," in Proc. IEEE Int. Behav. Model. Simulation Workshop, San Jose, CA, 2007, pp. 100-104.

[10] B. Razavi, "A study of injection locking and pulling in oscillators," IEEE J. Solid-State Circuits, vol. 39, no. 9, pp. 1415-1424, Sep. 2004.

[11] X. Lai and J. Roychowdhury, "Capturing oscillator injection locking via nonlinear phase-domain macromodels," IEEE Trans. Microw. Theory Tech., vol. 52, no. 9, pp. 2251-2261, Sep. 2004.

[12] X. Lai and J. Roychowdhury, "Automated oscillator macromodelling techniques for capturing amplitude variations and injection locking," in Proc. IEEE/ACM Int. Comput.-Aided Design Conf., San Jose, CA, 2004, pp. 687-694.

[13] P. Maffezzoni and D. D'Amore, "Evaluating pulling effects in oscillators due to small-signal injection," IEEE Trans. Comput.-Aided Design Integr. Circuits Syst., vol. 28, no. 1, pp. 22-31, Jan. 2009.

[14] C.-J. Li, C.-H. Hsiao, F.-K. Wang, T.-S. Horng, and K.-C. Peng, "A rigorous analysis of local oscillators pulling in frequency and discretetime domain," in Proc. IEEE Radio Freq. Integr. Circuits Symp. Dig., 2009, pp. 409-412.

[15] A. V. Oppenheim, R. W. Schafer, and J. R. Buck, Discrete-Time Signal Processing, 2nd ed. Upper Saddle River, NJ: Prentice-Hall, 1999.

[16] R. E. Best, Phase-Locked Loops: Theory, Design, and Applications, 2nd ed. New York: McGraw-Hill, 1993.

[17] K. Kurokawa, "Noise in synchronized oscillators," IEEE Trans. Microw. Theory Tech., vol. MTT-16, no. 4, pp. 234-240, Apr. 1968.

[18] M. E. Hines, J. R. Collinet, and J. G. Ondria, "FM noise suppression of an injection phase-locked oscillator," IEEE Trans. Microw. Theory Tech., vol. MTT-16, no. 9, pp. 738-742, Sep. 1968.

[19] T. Sugiura and S. Sugimoto, "FM noise reduction of Gunn-effect oscillators by injection locking," Proc. IEEE, vol. 57, no. 1, pp. 77-78, Jan. 1969. 
[20] J. R. Ashley, "Measured FM noise reduction by injection phase locking," Proc. IEEE, vol. 58, no. 1, pp. 155-157, Jan. 1970.

[21] K. F. Schünemann and K. Behm, "Nonlinear noise theory for synchronized oscillators," IEEE Trans. Microw. Theory Tech., vol. MTT-27, no. 5, pp. 452-458, Apr. 1979.

[22] J. C. Nallatamby, M. Prigent, J. C. Sarkissian, R. Quere, and J. Obregon, "A new approach to nonlinear analysis of noise behavior of synchronized oscillators and analog-frequency dividers," IEEE Trans. Microw. Theory Tech., vol. 46, no. 8, pp. 1168-1171, Aug. 1998.

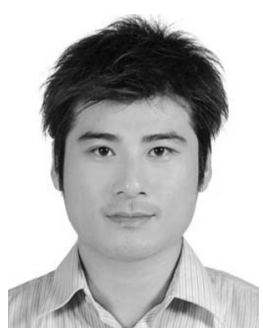

Chien-Jung Li (S'07-M'10) was born in Tainan, Taiwan, on October 26, 1979. He received the B.S.E.E. and Ph.D. degrees from National Sun Yat-Sen University, Kaohsiung, Taiwan, in 2002 and 2009, respectively.

$\mathrm{He}$ is currently a Postdoctoral Fellow with the Department of Electrical Engineering, National Sun Yat-Sen University. His research interests include power amplifier (PA) linearization techniques, frequency synthesizer designs, and LO pulling issues in direct-conversion transceivers.

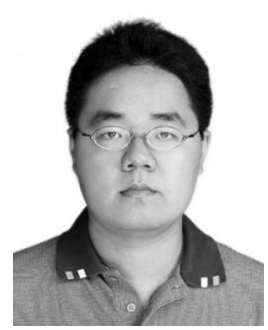

Chieh-Hsun Hsiao was born in Kaohsiung, Taiwan, on June 5, 1984. He received the B.S.E.E. and M.S.E.E. degrees from the National Sun Yat-Sen University, Kaohsiung, Taiwan, in 2006 and 2008, respectively, and is currently working toward the $\mathrm{Ph} . \mathrm{D}$. degree in electrical engineering at National Sun Yat-Sen University.

He is currently with the Department of Electrical Engineering, National Sun Yat-Sen University. His current research interests include PLOs and ILOs.

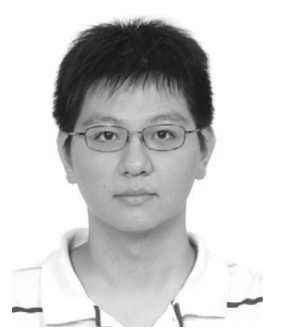

Fu-Kang Wang was born in Kaohsiung, Taiwan, on May 15, 1985. He received the B.S.E.E. and M.S.E.E. degrees from National Sun Yat-Sen University, Kaohsiung, Taiwan, in 2007 and 2009, respectively, and is currently working toward the $\mathrm{Ph} . \mathrm{D}$. degree in electrical engineering at National Sun Yat-Sen University.

He is currently with the Department of Electrical Engineering, National Sun Yat-Sen University. His current research interests include RF sensing techniques.

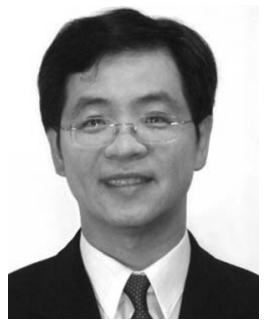

Tzyy-Sheng Horng (S'88-M'92-SM'05) was born in Taichung, Taiwan, on December 7, 1963. $\mathrm{He}$ received the B.S.E.E. degree from National Taiwan University, Taipei, Taiwan, in 1985, and the M.S.E.E. and Ph.D. degrees from the University of California at Los Angeles (UCLA), in 1990 and 1992, respectively.

Since August 1992, he has been with the Department of Electrical Engineering, National Sun Yat-Sen University, Kaohsiung, Taiwan, where he was the Director of the Telecommunication Research and Development Center (2003-2008) and Director of the Institute of Communications Engineering (2004-2007), and where he is currently a Professor. He has authored or coauthored over 100 technical publications published in refereed journals and conferences proceedings. He holds over ten patents. His research interests include RF and microwave integrated circuits and components, RF signal integrity for wireless system-in-package, and digitally assisted RF technologies.

Prof. Horng has served on several Technical Program Committees of international conferences including the International Association of Science and Technology for Development (IASTED) International Conference on Wireless and Optical Communications, the IEEE Region 10 International Technical Conference, the IEEE International Workshop on Electrical Design of Advanced Packaging and Systems, the Asia-Pacific Microwave Conference, and the IEEE Radio and Wireless Symposium. He was the recipient of the 1996 Young Scientist Award presented by the International Union of Radio Science, and the 1998 Industry-Education Cooperation Award presented by the Ministry of Education, Taiwan.

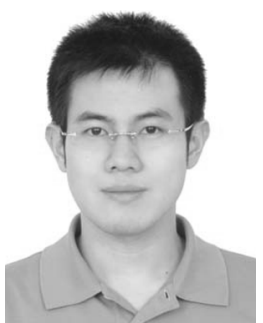

Kang-Chun Peng (S'00-M'05) was born in Taipei, Taiwan, on February 18, 1976. He received the B.S.E.E., M.S.E.E., and Ph.D. degrees from National Sun Yat-Sen University, Kaohsiung, Taiwan, in 1998, 2000, and 2005, respectively.

$\mathrm{He}$ is currently an Assistant Professor with the Department of Computer and Communication Engineering, National Kaohsiung First University of Science and Technology, Kaohsiung, Taiwan. His research interests include frequency synthesizers and RF transmitters. 